This document is confidential and is proprietary to the American Chemical Society and its authors. Do not copy or disclose without written permission. If you have received this item in error, notify the sender and delete all copies.

\title{
Crystal Structures of CaB3N3 at High Pressures
}

\begin{tabular}{|r|l|}
\hline Journal: & Inorganic Chemistry \\
\hline Manuscript ID & ic-2017-00904x.R2 \\
\hline Manuscript Type: & Article \\
\hline Date Submitted by the Author: & 26-May-2017 \\
\hline Complete List of Authors: & $\begin{array}{l}\text { Zhang, Miao; Beihua University; Center for High Pressure Science and } \\
\text { Technology Advanced Research } \\
\text { Guo, Ya-Nan; Jilin University, Department of Chemistry } \\
\text { Zhu, Li; Geophysical Laboratory, Carnegie Institution of Washington } \\
\text { Feng, Xiaolei; Jilin University } \\
\text { Redfern, Simon; University of Cambridge, Department of Earth Sciences; } \\
\text { Center for High Pressure Science and Technology Advanced Research } \\
\text { Chen, Jiuhua; Center for High Pressure Science and Technology Advanced } \\
\text { Research } \\
\text { Liu, Hanyu; Carnegie Institution of Washington, Geophysical laboratory } \\
\text { Tse, John; University of Saskatchewan, Physics and Engineering Physics; } \\
\text { Center for High Pressure Science and Technology Advanced Research }\end{array}$ \\
\hline \hline
\end{tabular}

SCHOLARONE

Manuscripts 


\title{
Crystal Structures of $\mathrm{CaB}_{3} \mathrm{~N}_{3}$ at High Pressures
}

\author{
Miao Zhang ${ }^{1,2, \dagger}$, Yanan Guo ${ }^{3, *},{ }^{\dagger}, \mathrm{Li} \mathrm{Zhu}^{4}$, Xiaolei Feng ${ }^{5}$, \\ Simon A. T. Redfern ${ }^{6,7}$, Jiuhua Chen ${ }^{2}$, Hanyu Liu $^{3} \&$ John S. Tse $2,5,8^{*}$ \\ ${ }^{1}$ College of Physics, Beihua University, Jilin 132013, China \\ ${ }^{2}$ Center for High Pressure Science and Technology Advanced Research, Jilin University, \\ Changchun 130015, China \\ ${ }^{3}$ State Key Laboratory of Inorganic Synthesis and Preparative Chemistry, College of Chemistry, \\ Jilin University, Changchun. 130012, China \\ ${ }^{4}$ Geophysical Laboratory, Carnegie Institution of Washington, Washington, D.C. 20015, United \\ States \\ ${ }^{5}$ State Key Laboratory of Superhard materials, Jilin University, Changchun. 130012, China \\ ${ }^{6}$ Department of Earth Sciences, University of Cambridge, Downing Street, Cambridge, CB2 3EQ, \\ $U K$ \\ ${ }^{7}$ Center for High Pressure Science and Technology Advanced Research, 1690 Cailun Rd, Pudong, \\ Shanghai, 201203, China \\ ${ }^{8}$ Department of Physics and Engineering Physics, University of Saskatchewan, Saskatoon, \\ Saskatchewan S7N 5E2, Canada
}




\begin{abstract}
Using global structure searches, we have explored the structural stability of $\mathrm{CaB}_{3} \mathrm{~N}_{3}$, a compound analogous to $\mathrm{CaC}_{6}$, under pressure. Two metastable high-pressure phases with space groups $R 3 c$ and $A m m 2$ were found to be stable between $29-42 \mathrm{GPa}$ and above $42 \mathrm{GPa}$, respectively. The two phases show different structural frameworks, analogous to graphitic $\mathrm{CaC}_{6}$. Phonon calculations confirm that both structures are also dynamically stable at high pressures. The electronic structure calculations show that the $R 3 c$ phase is a semiconductor with a band gap of $2.21 \mathrm{eV}$ and the $A m m 2$ phase is a semimetal. These findings help advance our understanding of the Ca-B-N ternary system.
\end{abstract}




\section{Introduction}

Since the discovery of superconductivity in alkali-metal intercalation compounds of graphite, graphite intercalation compounds (GICs) have been studied extensively. ${ }^{1-3}$ The GICs have ordered structures and are synthesized by inserting guest atoms or molecules between their hexagonal two-dimensional graphene sheets. ${ }^{1}$ Often, different intercalants lead to a series of compounds with regular stacking of $n$ graphite layers between two successive intercalant planes. Of course, it is also possible to intercalate three or even more layers of metals between two adjacent graphitic planes. Graphite is a semimetal, but the graphite in GICs, which is modified by electrons accepted or donated by the intercalant, may exhibit metallic or even superconducting behaviors. Exploration of GICs has resulted in notable success, for example, the superconducting transition temperature has been increased by almost two orders of magnitude to $11.5 \mathrm{~K}$ in $\mathrm{CaC}_{6}$, from that initially observed in $\mathrm{KC}_{8}\left(T_{\mathrm{c}}=0.15 \mathrm{~K}\right){ }^{4}$

It is known that boron nitride exists in a number of crystalline forms that are isoelectronic to carbon lattices with similar structural topologies. ${ }^{5}$ The hexagonal form of boron nitride $(\mathrm{hBN})$, similar to graphite, is the most stable and very compressible as compared to other BN polymorphs. Furthermore, the sphalerite-type form $(\mathrm{cBN})^{6}$ has a structure similar to cubic diamond and the rare wurtzite-type (wBN) is related to hexagonal diamond. ${ }^{7}$ The similarity between $\mathrm{BN}$ and carbon raises an interesting question: what happens if the carbon in GICs is replaced by B and N atoms? For example, if the graphitic carbon is replaced by $\mathrm{BN}$ in $\mathrm{CaC}_{6}$, will the resulting $\mathrm{CaB}_{3} \mathrm{~N}_{3}$ show the same structural characteristics of GICs, or contain $\mathrm{BN}_{2}{ }^{3-}$ anions as in the experimentally synthesized $\mathrm{Ca}_{3}\left(\mathrm{BN}_{2}\right)_{2}$ and $\mathrm{Ca}_{3} \mathrm{BN}_{3}$ compounds? ${ }^{8,9}$ Does it possess novel physical and chemical properties? These questions are addressed here.

In this work, possible high-pressure phases of $\mathrm{CaB}_{3} \mathrm{~N}_{3}$ are explored by first-principles computational methods using the particle swarm optimization algorithm for crystal structure prediction. ${ }^{10,11}$ The particle swarm optimization implemented in the CALYPSO code has shown to have reliably predicted crystal structures for a large variety of chemical systems under ambient and high pressure 
conditions. ${ }^{12-18}$ In this study, two new high-pressure structures of $\mathrm{CaB}_{3} \mathrm{~N}_{3}$ with space group $R 3 c$ and Amm2, which are stable at 29-42 GPa and above $42 \mathrm{GPa}$, respectively, were found. Furthermore, electronic structure calculations show that the $R 3 c$ structure is a semiconductor while the Amm2 structure is a semimetal. The present results suggest $\mathrm{CaB}_{3} \mathrm{~N}_{3}$ undergoes a structural phase transition from semiconductor to semimetal at approximately $42 \mathrm{GPa}$. In addition, phonon calculations show both structures are dynamically stable at high pressure and can be recovered under normal pressure.

\section{Computational details}

Structure searches for $\mathrm{CaB}_{3} \mathrm{~N}_{3}$ were performed with the swarm intelligence-based CALYPSO code. ${ }^{10,11}$ The CALYPSO code is a useful tool to predict the crystal structures of materials. ${ }^{19-22}$ Structural relaxations were performed using density functional theory (DFT) within the Perdew-Burke-Ernzerhof (PBE) ${ }^{23}$ parameterization of the generalized gradient approximation (GGA), as implemented in the Vienna ab initio simulation package (VASP) code. ${ }^{24}$ The all-electron projector augmented wave (PAW) potentials ${ }^{25}$ were used in which the $3 s^{2} 3 p^{6} 4 s^{2}, 2 s^{2} 2 p^{1}$ and $2 s^{2} 2 p^{3}$ are treated as valence electrons for the $\mathrm{Ca}, \mathrm{B}$ and $\mathrm{N}$ atoms, respectively. For the lowest enthalpy structures, more refined calculations were performed using an energy cut of $700 \mathrm{eV}$ and dense Monkhorst $k$-meshes ${ }^{26}$ to ensure the enthalpy calculations were well-converged (A $k$-mesh of $8 \times 8 \times 12$ is used for the Amm2 structure and a $k$-mesh of $10 \times 10 \times 10$ is used for the $R 3 c$ structure). The absence of negative phonon frequencies in a crystal is the definitive indication of the structural stability. We employed the supercell approach to compute the interplanar force constants required for the calculations of phonon dispersion curves, which were computed from finite displacements of the atoms according to the crystal symmetry. The Hellmann-Feynman forces and total energies were calculated. Once the force constant is determined, the phonon frequency at selected $q$ points along the symmetry lines in the Brillouin zone can be calculated. Phonon calculations were calculated using the 
PHONOPY $\operatorname{code}^{27}$ using a $2 \times 2 \times 2$ supercell. The elastic moduli were determined from the stress-strain relationships. The bulk modulus and shear modulus were estimated from the Voigt-Reuss-Hill approximation. ${ }^{28}$ The structures are plotted using VESTA. $^{29}$

\section{Results and discussions}

We have performed structure prediction simulations in the pressure range of 0-100 $\mathrm{GPa}$ on model systems consisted of one, two, three and four $\mathrm{CaB}_{3} \mathrm{~N}_{3}$ formula units. The thermodynamic phase stabilities at high pressure were determined from the calculated enthalpy of formation $H_{\mathrm{f}}$ of the predicted $\mathrm{CaB}_{3} \mathrm{~N}_{3}$ compounds with respect to the decomposition into $\mathrm{Ca}$ and $\mathrm{BN}$ according to the following expression:

$$
H_{\mathrm{f}}\left[\mathrm{CaB}_{3} \mathrm{~N}_{3}\right]=H\left[\mathrm{CaB}_{3} \mathrm{~N}_{3}\right]-H(\mathrm{Ca})-3 H(\mathrm{BN})
$$

where $H\left[\mathrm{CaB}_{3} \mathrm{~N}_{3}\right]$ is the enthalpy of $\mathrm{CaB}_{3} \mathrm{~N}_{3}, H(\mathrm{Ca})$ is the enthalpy of elemental $\mathrm{Ca}$, and $H(\mathrm{BN})$ is the enthalpy of $\mathrm{BN}$. The structure of $\mathrm{Ca}$ was assumed to be fcc at 0 19.5 GPa, bcc at 19.5 - $32 \mathrm{GPa}$ and simple cubic above $32 \mathrm{GPa}$. For BN, the hexagon structure (0-4 GPa) and the cubic BN structure (above $4 \mathrm{GPa}$ ) was adopted at different pressures.

The PSO searches found many high-pressure structures with two particular phases stable over different pressure ranges. Above $\sim 29 \mathrm{GPa}$, a low energy hexagonal $R 3 \mathrm{c}$ phase is found to be more stable than an equivalent mixture of elemental $\mathrm{Ca}$ and $\mathrm{cBN}$. Upon increasing pressure to $\sim 42 \mathrm{GPa}$, an orthorhombic Amm2 structure becomes energetically more favorable than the $R 3 c$ structure. [Figure 1]. We have also calculated the enthalpy of GIC-type $\mathrm{CaB}_{3} \mathrm{~N}_{3}$ structure from 0 to $100 \mathrm{GPa}$, as shown in Figure 1a. The results show that the enthalpy of the GIC-type structure is higher than predicted $A m m 2$ and $R 3 c$ structures. We have studied the finite temperature phase diagram of $\mathrm{CaB}_{3} \mathrm{~N}_{3}$ based on the quasi-harmonic approximation and the results are shown in Figure 1b. 

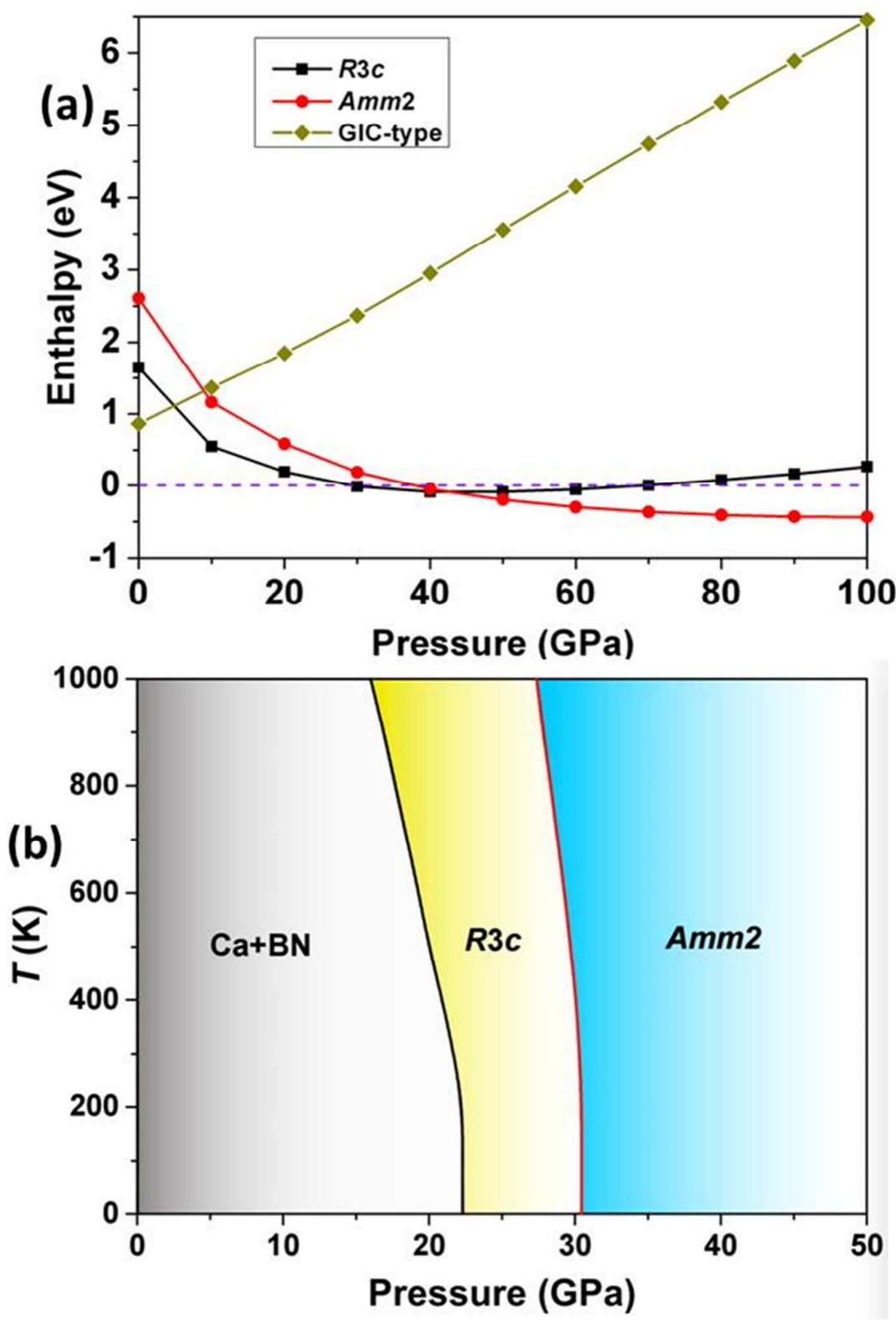

Figure 1. (a) The calculated enthalpy of formation per formula unit as function of pressure relative to decomposition into $\mathrm{Ca}, \mathrm{BN}, \mathrm{N}$ with their lowest structure at different pressures. (b) Phase diagram of $\mathrm{CaB}_{3} \mathrm{~N}_{3}$ based on the quasi-harmonic approximation.

The $R 3 c$ structure is constructed from rings of boron and nitrogen atoms with $\mathrm{Ca}$ atoms located in the middle of the channels [Figure 2a-c]. The structure is distinctly different to the graphitic $\mathrm{CaC}_{6}$. In this structure, both $\mathrm{B}$ and $\mathrm{N}$ atoms are threefold coordinated [Figure 2c] with B-N bond lengths of $1.536 \AA$ and $1.504 \AA$ and the shortest Ca-Ca distance is $4.071 \AA$. 
(a)

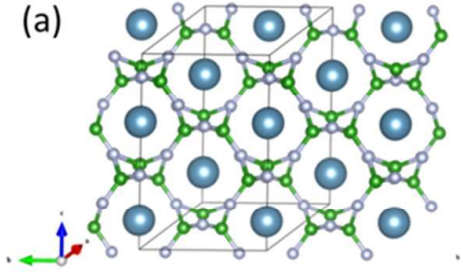

q

(b)

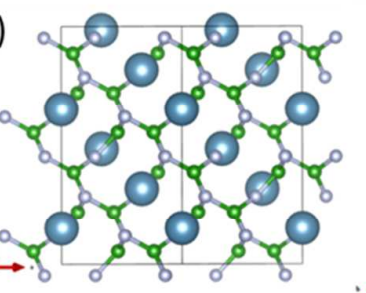

(c)

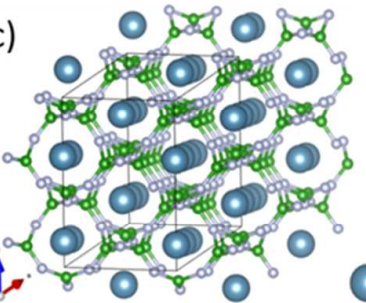

$\odot \mathrm{Ca}$

(d)

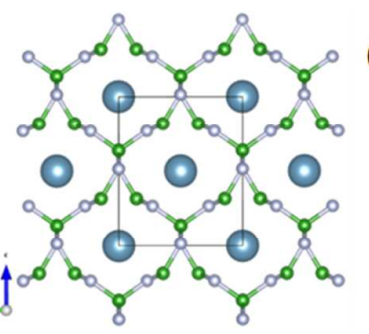

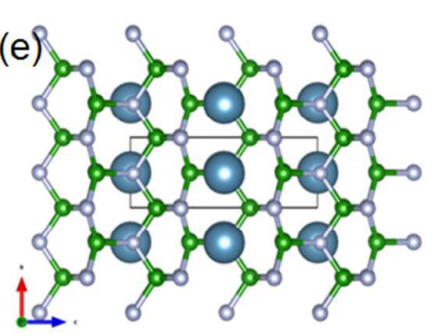

- $\mathrm{B}$

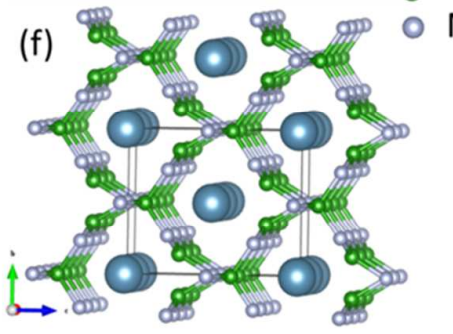

Figure 2. Crystal structures of $R 3 c$ structure (a)-(c) and (d)-(e) Amm 2 structure.

In the Amm 2 structure, the $\mathrm{B}$ and $\mathrm{N}$ atoms are three- and four-fold coordinated [Figure 2d-f]. The B-N bond lengths of B-N at the threefold B and $\mathrm{N}$ coordinated sites are $1.566 \AA$ and 1.623. In comparison, the B-N lengths at the fourfold coordinated B and $\mathrm{N}$ sites are $1.586 \AA$ and $1.635 \AA$, only slightly longer than those in threefold coordination. The most remarkable observation is that the shortest $\mathrm{Ca}-\mathrm{Ca}$ distance in Amm2 structure at ambient pressure is $2.638 \AA$. This is much shorter than that in the $R 3 c$ structure. The very short $\mathrm{Ca}$...Ca contact is indicative of almost full transfer of valence electrons to the $\mathrm{BN}$ framework, since the $\mathrm{Ca}^{2+}$ ionic radius is $1.17 \AA$ which results in a $\mathrm{Ca}-\mathrm{Ca}$ distance comparable to $2.638 \AA$ in the Amm2 phase. This phenomenon has already been reported in the earlier study of high pressure $\mathrm{K}-\mathrm{Ag}$ alloys. $^{30}$ 

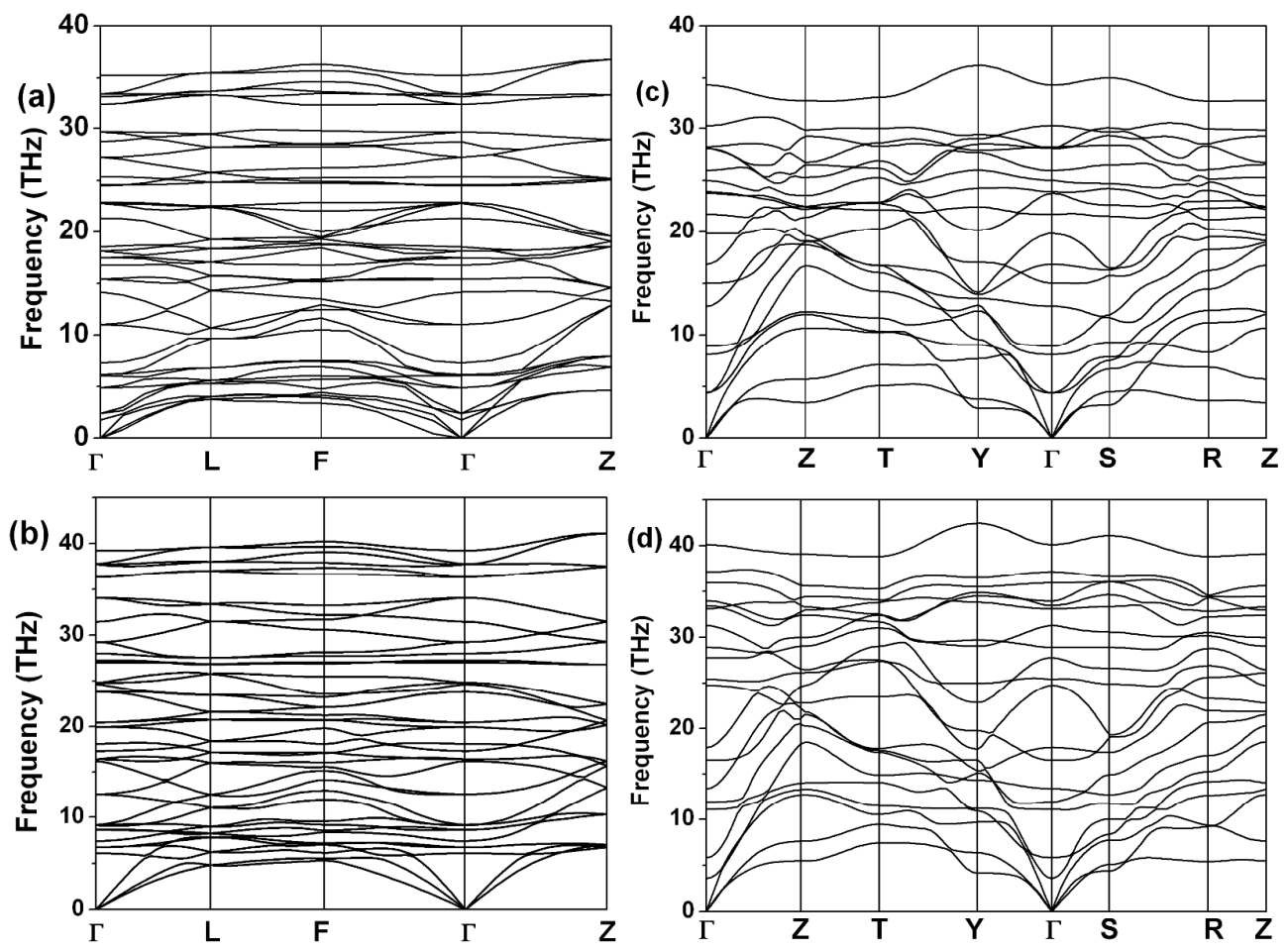

Figure. 3 The calculated phonon dispersions of (a) and (b) the $R 3 c$ structure at 0 and $30 \mathrm{GPa}$ and (c) and (d) the Amm2 structure at 0 and $50 \mathrm{GPa}$.

Figures 3 (a) and 3(b) show the phonon dispersion relationships of the $R 3 c$ structure at $0 \mathrm{GPa}$ and $30 \mathrm{GPa}$. Fig. 3 (c) and 3 (d) show that of Amm2 structure at 0 $\mathrm{GPa}$ and $50 \mathrm{GPa}$, respectively. In both cases, no negative (imaginary) frequencies were observed in the Brillouin zone, indicating that the structures are dynamically stable at ambient pressure and across the corresponding stable pressure ranges. The observation suggests that both structures may be quench-recoverable at low temperature as long as the activation barriers to decomposition are reasonably high. 

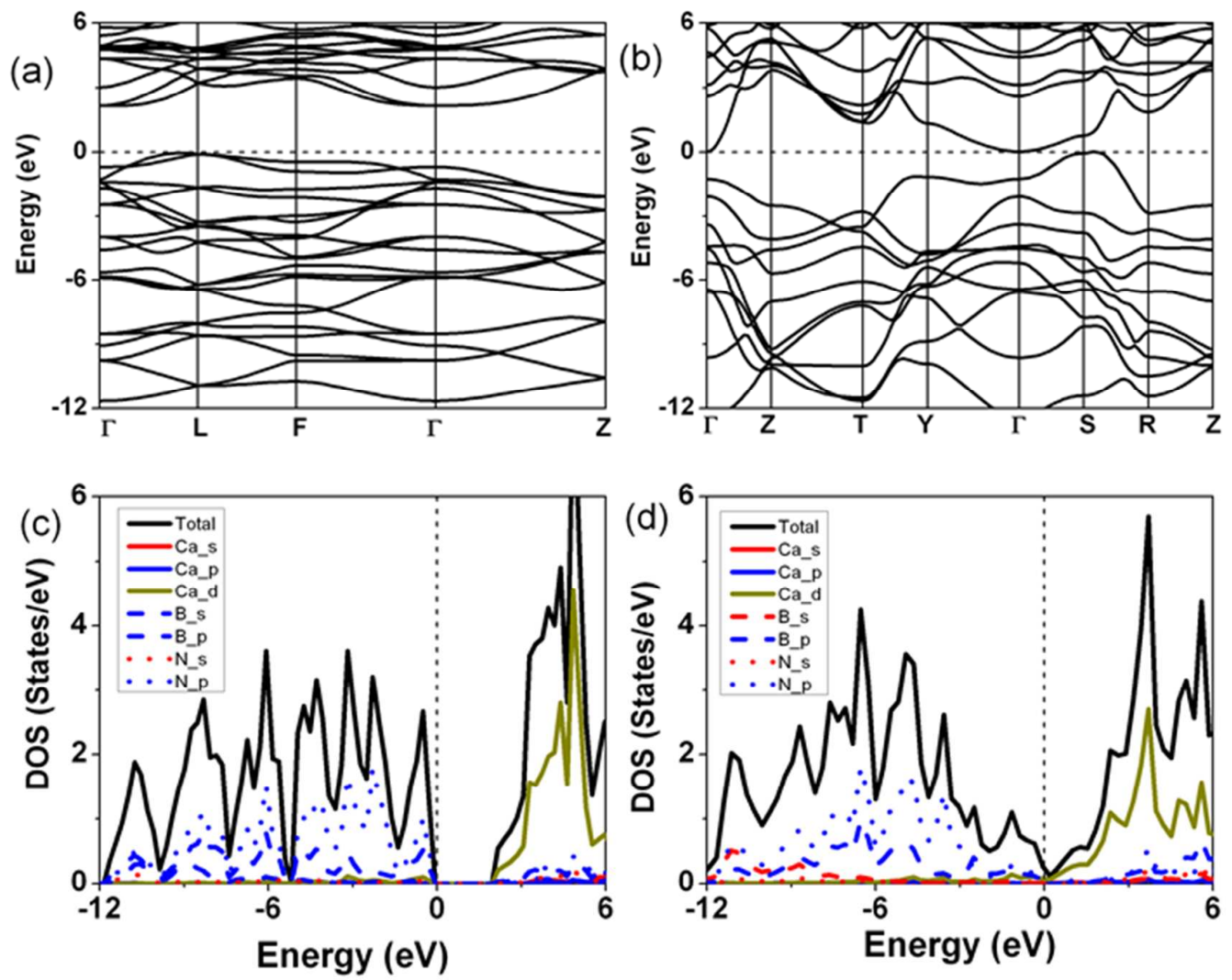

Figure. 4 The calculated electronic band structures of (a) the $R 3 c$ structure and (b) the $A m m 2$ structure, and electronic density of states of (c) the $R 3 c$ structure and (d) the Amm2 structure.

Figure 4shows the calculated electronic band structures of the $R 3 c$ (4a) and Amm2 (4b) phase of $\mathrm{CaB}_{3} \mathrm{~N}_{3}$ at $0 \mathrm{GPa}$. The zero energy refers to the top of valence bands. As shown in Figure 4(a), the $R 3 c$ structure is a semiconductor with an estimated indirect band gap of $2.21 \mathrm{eV}$. It is found that the maximum valence band located at $\mathrm{L}$ point and the minimum conduction band located at $\Gamma$ point. The calculated electronic density of states [Figure 4 (c) and 4(d)] show substantial overlap of the B-p and N-pbands, indicating strong covalent $\mathrm{B}-\mathrm{N}$ bonding in both the $R 3 \mathrm{c}$ and $A m m 2$. According to these calculations the higher-pressure Amm2 structure appears to be a semimetal. We should point out DFT methods typically underestimate the band gap, however. ${ }^{31,32}$ To gain a more detailed insight into the bonding character, the electronic localization functions (ELF) were calculated. The 3D ELF iso-surfaces of the $R 3 \mathrm{c}$ and $A m m 2$ phases at ambient pressure are displayed in Figure 5a and 5b, respectively. The large 
value of ELF between the $\mathrm{B}$ and $\mathrm{N}$ atoms show significant covalent (two-electron pair) character of the B-N bonds. Moreover, as can be seen in Figure 5a and 5b, there are large regions of high ELF (0.85) close to B atoms. This suggested the presence of electrides in $\mathrm{CaB}_{3} \mathrm{~N}_{3}$ at high pressures. The results are consistent with previous studies. $^{33,34}$ We also performed crystal orbital Hamiltonian population (COHP) analysis by projecting the plane wave orbitals to atomic basis sets. ${ }^{35}$ The negative COHPs (Fig. 5c and 5d) below the Fermi level show ambiguously covalent bonding between $\mathrm{B}$ and $\mathrm{N}$ atoms.
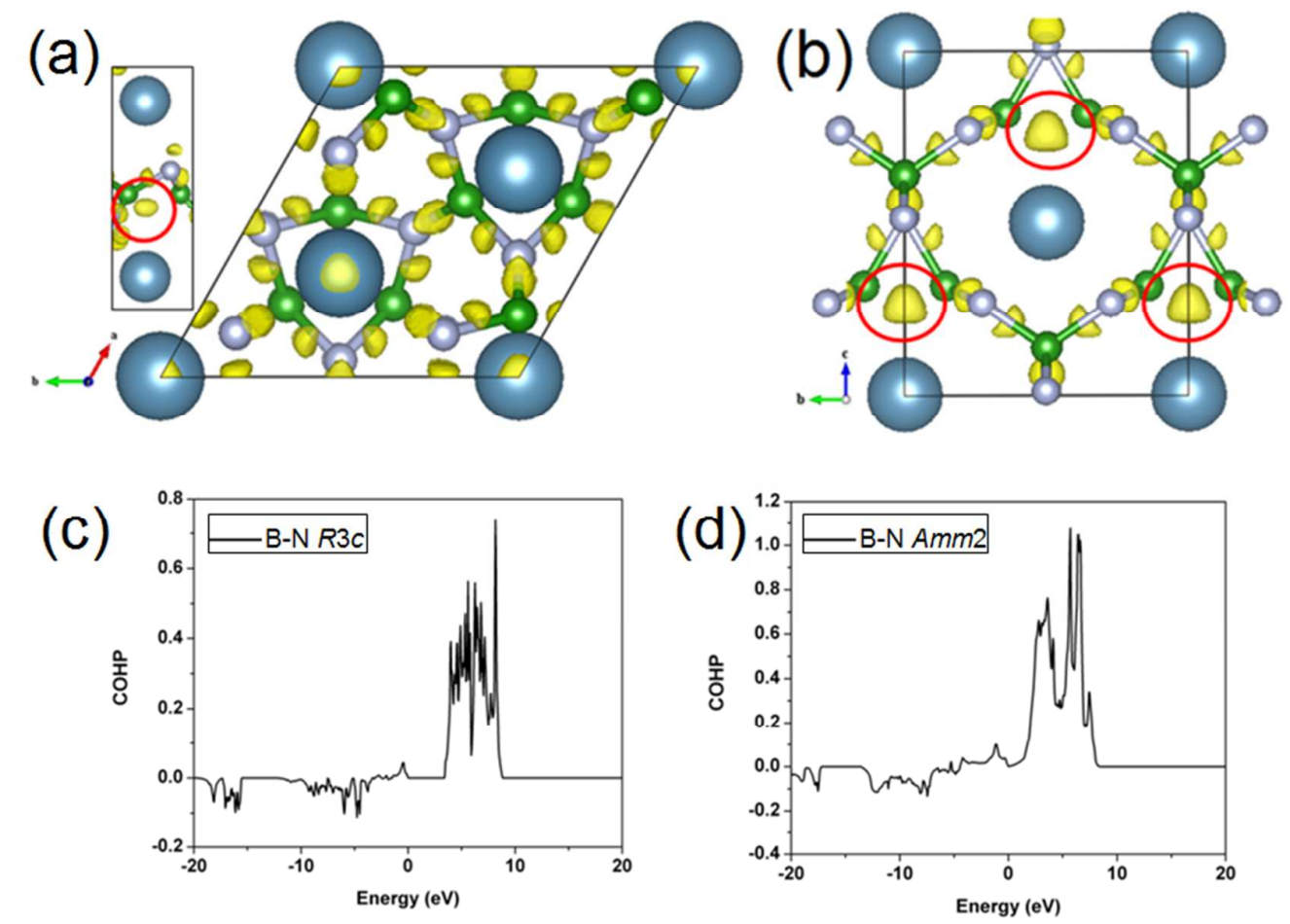

Figure.5 Isosurface of ELF for $R 3 c$ (a) and Amm2 (b) with the value of 0.85 . The Crystal orbital Hamiltonian population (COHP) for pairs of B-N in $R 3 c$ (c) and Amm2 (d).

Table 1, The calculated elastic constants $C_{\mathrm{ij}}(\mathrm{GPa})$, bulk modulus $\left(B_{0}\right)$, shear modulus $(G)$, Young's modulus $(Y)$, Poisson's ratio $v$, and Vickers hardness $\left(H_{\mathrm{v}}\right)$ for $R 3 c$ structure and $A m m 2$ structure.

\begin{tabular}{|c|c|c|c|c|c|c|c|c|c|c|c|c|c|}
\hline & $C_{11}$ & $C_{12}$ & $C_{13}$ & $C_{22}$ & $C_{23}$ & $C_{33}$ & $C_{44}$ & $C_{55}$ & $C_{66}$ & $B_{0}$ & $G$ & $Y$ & $v$ \\
\hline$R 3 c$ & 381 & 107 & 153 & & & 346 & 191 & & 137 & 215 & 144 & 354 & 0.2254 \\
\hline Amm2 & 685 & 74 & 48 & 499 & 136 & 472 & 205 & 179 & 244 & 240 & 214 & 495 & 0.1561 \\
\hline
\end{tabular}

The mechanical properties (elastic constants, anisotropy, and hardness) of the 
predicted $R 3 \mathrm{c}$ and $A m m 2$ structures are important for their potential technological and industrial applications. The elastic constants, bulk modulus, shear modulus, Young's modulus and Poisson's ratio of the $R 3 c$ structure and $A m m 2$ structures are summarized in Table I. The elastic constants for both structures were calculated by the strain-stress method. For the rhombohderal crystals, the mechanical stability requires the elastic constants satisfying the mechanical criteria: $\left.\left.C_{11}\right\rangle\left|C_{12}\right|, 2 C_{13}{ }^{2}<C_{33}\left(C_{11}+C_{12}\right), C_{44}\right\rangle$ $0, C_{66}>0$. We can see that the elastic constants for the $R 3 c$ phase satisfy the above conditions, implying that this phase is elastically stable at ambient pressure. For the orthorhombic Amm2 phase, tests on the elastic stability criterion also show that the elastic constants satisfy the conditions: $C_{11}>0, C_{11} C_{22}>C_{13}{ }^{2},\left[C_{11} C_{22} C_{33}+\right.$ $2 C_{12} C_{13} C_{23}-C_{11} C_{13}^{2}-\mathrm{C}_{22} C_{13}^{2}-C_{33} C_{13}^{2}>0, C_{44}>0, C_{55}>0, C_{66}>0$, and hence the calculations indicate that this phase is elastically stable. It is noteworthy that the bulk modulus calculated from the elastic constants for the Amm2 phase is $240 \mathrm{GPa}$ (Table1), which implies it is a high compressibility material. The ratios of the shear $(G)$ and bulk modulus $(B), G / B$, are 0.67 and 0.89 for the $R 3 c$ and $A m m 2$ phases at 0 $\mathrm{GPa}$, respectively. These values are similar to those of other known superhard materials (0.9-1.2), such as diamond and $\mathrm{C}_{3} \mathrm{~N}_{4}$. Therefore, these $\mathrm{CaB}_{3} \mathrm{~N}_{3}$ polymoprhs are likely be potential hard materials. The hardness is similar to that previously reported for some nitrides. ${ }^{36}$

\section{Conclusions}

In summary, two novel high-pressure phases of $\mathrm{CaB}_{3} \mathrm{~N}_{3}$, rhombohedral $R 3 c$ and orthorhombic Amm2, were found to be metastable between 29-42 GPa and above 42 $\mathrm{GPa}$, respectively. Although in the natural state BN exists in forms similar to its carbon analogs, at high pressures $\mathrm{CaB}_{3} \mathrm{~N}_{3}$ forms $3 \mathrm{D}$ networks which differ from $\mathrm{CaC}_{6}$. Theoretical phonon band structures confirm that both structures are dynamically stable at ambient and high pressures. The electronic structure of the $R 3 c$ phase shows that it is a semiconductor with a band gap of $2.21 \mathrm{eV}$. In contrast, the higher pressure Amm2 structure appears to be a semimetal. This suggests that $\mathrm{CaB}_{3} \mathrm{~N}_{3}$ will undergo both structural and semiconductor-semimetal phase transitions at high pressure. 


\title{
Supporting information:
}

Table S1-S3 and Figure S1.

\author{
Author information: \\ Corresponding author: yananguo813@gmail.com or john.tse@usask.ca \\ ${ }^{\dagger}$ M. Z and Y. G. are contributed equally to this work.
}

\section{Acknowledgements}

This research was supported by the Natural Science Foundation of China under No. 11504007, 11404035, and the Scientific and Technological Research Project of the “13th Five-Year Plan” of Jilin Provincial Education Department under Grant No. 201648 and 201649. Work at Carnegie was supported by EFree, an Energy Frontier Research Center funded by the DOE, Office of Science, Basic Energy Sciences under Award No. DE-SC-0001057. The infrastructure and facilities used at Carnegie were supported by NNSA Grant No. DE-NA-0002006, CDAC. JST thanks the National Natural Science Foundation of China under Grant No. 11474126. 


\section{References:}

1. Weller, T. E.; Ellerby, M.; Saxena, S. S.; Smith, R. P.; Skipper, N. T., Superconductivity in the intercalated graphite compounds $\mathrm{C}_{6} \mathrm{Yb}$ and $\mathrm{C}_{6} \mathrm{Ca}$. Nat. Phys. 2005, 1, 39-41.

2. Mazin, I., Intercalant-driven superconductivity in $\mathrm{YbC}_{6}$ and $\mathrm{CaC}_{6}$. Phys. Rev. Lett. 2005, 95, 227001.

3. Emery, N.; Hérold, C.; d'Asturo, M.; Garcia, V.; Bellin, C.; Marêché, J. F.; Lagrange, P.; Loupias, G., Superconductivity of bulk $\mathrm{CaC}_{6}$. Phys. Rev. Lett. 2005, 95, 087003.

4. Hannay, N.; Geballe, T.; Matthias, B.; Andres, K.; Schmidt, P.; MacNair, D., Superconductivity in graphitic compounds. Phys. Rev. Lett. 1965, 14, 225.

5. Paine, R. T.; Narula, C. K., Synthetic routes to boron nitride. Chem. Rev. 1990, 90, 73-91.

6. Mirkarimi, P.; McCarty, K.; Medlin, D., Review of advances in cubic boron nitride film synthesis. Mater. Sci. Eng. R. Rep. 1997, 21, 47-100.

7. Bundy, F.; Kasper, J., Hexagonal diamond: a new form of carbon. J. Chem. Phys. 1967, 46, 3437-3446.

8. Haberlen, M.; Glaser, J.; Meyer, H.-J., Phase transitions in $\mathrm{Ca}_{3}\left(\mathrm{BN}_{2}\right)_{2}$ and $\mathrm{Sr}_{3}\left(\mathrm{BN}_{2}\right)_{2}$. Journal of Soli. Stat. Chem. 2005, 178, 1478-1487.

9. Haberlen, M.; Glaser, J.; Meyer, $\mathrm{H}_{.}, \mathrm{Ca}_{3}\left(\mathrm{BN}_{2}\right) \mathrm{N}$, the missing compound in the quasi binary Ca3N2-BN system. Zeit. Anor. Allg. Chem. 2002, 628, 1959-1962.

10. Wang, Y.; Lv, J.; Zhu, L.; Ma, Y., CALYPSO: A method for crystal structure prediction. Computer Phys. Commun. 2012, 183, 2063-2070.

11. Wang, Y.; Lv, J.; Zhu, L.; Ma, Y., Crystal structure prediction via particle-swarm optimization. Phys. Rev. B 2010, 82, 094116.

12. Wang, Y.; Liu, H.; Lv, J.; Zhu, L.; Wang, H.; Ma, Y., High pressure partially ionic phase of water ice. Nat. Commun. 2011, 2, 563.

13. Zhu, L.; wang, H.; Wang, Y.; Lv, J.; Ma, Y.; Cui, Q.; Ma, Y.; Zou, G., Substitutional Alloy of Bi and Te at High Pressure. Phys. Rev. Lett. 2011, 106, 145501.

14. Miao, M.-s., Caesium in high oxidation states and as a p-block element. Nat. Chem. 2013, 5, 846-852.

15. Zhu, L.; Liu, H.; Pickard, C. J.; Zou, G.; Ma, Y., Reactions of xenon with iron and nickel are predicted in the Earth's inner core. Nat. Chem. 2014, 6, 644-648.

16. Yong, X.; Liu, H.; Wu, M.; Yao, Y.; John, S. T.; Dias, R.; Yoo, C.-S., Crystal structures and dynamical properties of dense $\mathrm{CO}_{2}$. Proc. Nati. Acad. Sci. USA 2016, 113, 11110-11115

17. Liu, H.; Naumov, I. I.; Hemley, R. J., Dense Hydrocarbon Structures at Megabar Pressures. J. Phys. Chem. Lett. 2016, 7, 4218-4222.

18. Shi, J.; Cui, W.; Flores-Livas, J. A.; San-Miguel, A.; Botti, S.; Marques, M. A., Investigation of new phases in the Ba-Si phase diagram under high pressure using ab initio structural search. Phys. Chem. Chem. Phys. 2016, 18, 8108-8114.

19. Liu, H.; Wang, H.; Ma, Y., Quasi-Molecular and Atomic Phases of Dense Solid Hydrogen. J. Phys. Chem. C 2012, 116, 9221-9226.

20. Liu, H.; Yao, Y.; Klug, D. D., Stable structures of $\mathrm{He}$ and $\mathrm{H}_{2} \mathrm{O}$ at high pressure. Phys. Rev. B 2015, 91, 014102.

21. Zhang, M.; Liu, H.; Li, Q.; Gao, B.; Wang, Y.; Li, H.; Chen, C.; Ma, Y., Superhard $\mathrm{BC}_{3}$ in cubic diamond structure. Phys. Rev. Lett. 2015, 114, 015502.

22. Li, Y.; Hao, J.; Liu, H.; Lu, S.; John, S. T., High-Energy Density and Superhard Nitrogen-Rich BN 
Compounds. Phys. Rev. Lett. 2015, 115, 105502.

23. Perdew, J. P.; Burke, K.; Ernzerhof, M., Generalized Gradient Approximation Made Simple. Phys. Rev. Lett. 1996, 77, 3865-3868.

24. Kresse, G.; Furthmuller, J., Efficient iterative schemes for ab initio total-energy calculations using a plane-wave basis set. Phys. Rev. B 1996, 54, 11169.

25. Kresse, G.; Joubert, D., From ultrasoft pseudopotentials to the projector augmented-wave method. Phys. Rev. B 1999, 59, 1758.

26. Monkhorst, H. J.; Pack, J. D., Special points for Brillouin-zone integrations. Phys. Rev. B 1976, 13, 5188.

27. Togo, A.; Oba, F.; Tanaka, I., First-principles calculations of the ferroelastic transition between rutile-type and $\mathrm{CaCl}_{2}$-type $\mathrm{SiO}_{2}$ at high pressures. Phys. Rev. B 2008, 78, 134106.

28. Zhang, M.; Lu, M.; Du, Y.; Gao, L.; Lu, C.; Liu, H., Hardness of FeB F $_{4}$ Density functional theory investigation. J. Chem. Phys. 2014, 140, 174505.

29. Momma, K.; Izumi, F., VESTA: a three-dimensional visualization system for electronic and structural analysis. J. Appl. Crys. 2008, 41, 653-658.

30. Tse, J.; Frapper, G.; Ker, A.; Rousseau, R.; Klug, D., Phase stability and electronic structure of K-Ag intermetallics at high pressure. Phys. Rev. Lett. 1999, 82, 4472.

31. Lopez-Moreno, S.; Rodriguez-Hernandez, P.; Munoz, A.; Romero, A.; Errandonea, D., First-principles calculations of electronic, vibrational, and structural properties of scheelite EuWO under pressure. Phys. Rev. B 2011, 84, 064108.

32. Ruiz-Fuertes, J.; Lopez-Moreno, S.; Lopez-Solano, J.; Errandonea, D.; Segura, A.; Lacomba-Perales, R.; Munoz, A.; Radescu, S.; Rodriguez-Hernandez, P.; Gospodinov, M., Pressure effects on the electronic and optical properties of $\mathrm{A} \mathrm{WO}_{4}$ wolframites $(\mathrm{A}=\mathrm{Cd}, \mathrm{Mg}, \mathrm{Mn}$, and $\mathrm{Zn})$ : The distinctive behavior of multiferroic $\mathrm{MnWO}_{4}$. Phys. Rev. B 2012, 86, 125202.

33. Miao, M.-S.; Hoffmann, R., High pressure electrides: a predictive chemical and physical theory. Acc. Chem. Res. 2014, 47, 1311-1317.

34. Miao, M.-s.; Hoffmann, R., High-pressure electrides: the chemical nature of interstitial quasiatoms. J. Amer. Chem. Soc. 2015, 137, 3631-3637.

35. Dronskowski, R.; Bloechl, P. E., Crystal Orbital Hamilton Populations (COHP). energy-resolved visualization of chemical bonding in solids based on density-functional calculations. J. Phys. Chem. 1993, 97, 8617-8624.

36. Errandonea, D.; Ferrer-Roca, C.; Martinez-Garcia, D.; Segura, A.; Gomis, O.; Munoz, A.; Rodriguez-Hernandez, P.; Lopez-Solano, J.; Alconchel, S.; Sapina, F., High-pressure x-ray diffraction and ab initio study of $\mathrm{Ni}_{2} \mathrm{Mo}_{3} \mathrm{~N}, \mathrm{Pd}_{2} \mathrm{Mo}_{3} \mathrm{~N}, \mathrm{Pt}_{2} \mathrm{Mo}_{3} \mathrm{~N}, \mathrm{Co}_{3} \mathrm{Mo}_{3} \mathrm{~N}$, and $\mathrm{Fe}_{3} \mathrm{Mo}_{3} \mathrm{~N}$ : Two families of ultra-incompressible bimetallic interstitial nitrides. Phys. Rev. B 2010, 82, 174105. 


\section{For Table of Contents Only}

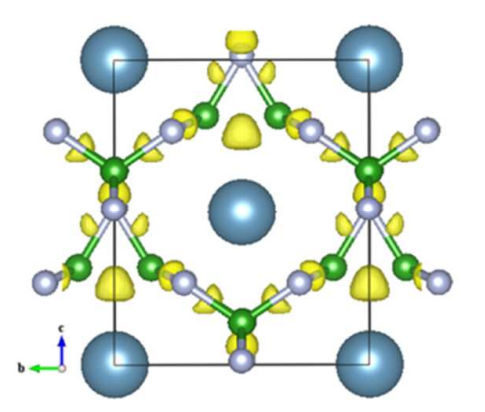

We have explored the structural stability of $\mathrm{CaB}_{3} \mathrm{~N}_{3}$, a compound analogous to $\mathrm{CaC}_{6}$, under pressure. Two metastable high-pressure phases were found to be stable. The two phases show different structural frameworks, analogous to graphitic $\mathrm{CaC}_{6}$. Phonon calculations confirm that both structures are also dynamically stable at high pressures. These findings help advance our understanding of the Ca-B-N ternary system. 